\title{
"Aging" of the structure of crystals of hard colloidal spheres
}

\author{
Willem K. Kegel ${ }^{\mathrm{a})}$ and Jan K. G. Dhont \\ Van't Hoff Laboratory for Physical and Colloid Chemistry, Debye Institute, Utrecht University, \\ Padualaan 8, 3584 CH Utrecht, The Netherlands
}

(Received 4 October 1999; accepted 19 November 1999)

\begin{abstract}
We study the development of the structure of crystals of colloidal hard spheres in time when gravity effects are minimal and polydispersity is small $(<3 \%)$. The initial stacking of the close-packed hexagonal layers that make up the crystals is varied by applying various types of shear stress during nucleation of the crystals. The experimental powder diffraction patterns are consistent with a fraction of a faulted-twinned face-centered cubic (fcc) structure that grows at the expense of randomly stacked crystallites. If a faulted-twinned fcc structure is generated initially, no change is found over a considerable time. The present observations rule out the possibility that a randomly stacked structure is the equilibrium structure of colloidal crystals of (nearly) hard spheres, and point to the thermodynamic or kinetic stability of faulted-twinned fcc crystals in these systems. (C) 2000 American Institute of Physics. [S0021-9606(00)70307-2]
\end{abstract}

\section{INTRODUCTION}

A collection of hard spheres under thermal agitation is probably the simplest model of interacting atoms. Computer simulations reveal that these systems have a true freezing transition $^{1,2}$ at a density that is significantly smaller than the close-packed density of spheres. This transition is driven by entropy. Under appropriate conditions, sterically stabilized colloidal suspensions are an experimental manifestation of hard spheres, ${ }^{3}$ where Brownian displacements play an equivalent role as thermal movement in atomic systems. In colloidal suspensions, the particles are stabilized by a relatively thin layer of polymers attached to their surface, and are dispersed in a solvent with the same refractive index as the particles so as to minimize van der Waals attraction. In such a system, the freezing transition of hard spheres was indeed verified: ${ }^{3}$ at volume fractions smaller than $\varphi=0.494$ (the freezing volume fraction) the system is in a fluid state. At $0.494<\varphi<0.545$, crystals of hard spheres coexist with colloidal fluid, and at $\varphi>0.545$ (the melting volume fraction), a single (crystalline) solid phase is found. In this work we shall be concerned with the structure of crystals of colloidal hard spheres.

The structure of spheres that occupies the least space is a stacking of close-packed hexagonal layers. ${ }^{4}$ Within a crystal, each layer can adopt one out of three possible lateral positions, $A, B$, and $C$. Close packing is assured if two adjacent layers have different lateral positions, but if layer $n$ has the reference position $A$ and $(n+1)$ has $B$, then layer $(n+2)$ can have either $C$ or $A$. If the first sequence is extended over the whole crystal, one has a face centered cubic (fcc) structure, and the extension of the second possibility leads to hexagonal close-packed (hcp) crystals. Stacking faults lead to structures that are neither fcc nor hcp. Defining $\alpha$ as the probability to find a layer at $(n+2)$ that has a different position from the one at $n$, one has fcc if $\alpha=1$, hcp if $\alpha=0$, and a completely random stacking (also referred to as rhcp, random

a)Electronic mail: W.K.Kegel@chem.uu.nl hexagonal close packing) if $\alpha=0.5$. Most close-packed atomic crystals have either perfect fcc or hcp, an exception being cobalt, which has $\alpha=0.1$, as shown by Wilson. ${ }^{5}$ About a decade ago, Pusey et $a l .{ }^{6}$ reported light-scattering measurements of powder diffraction patterns of crystals of hard colloidal spheres. Their results are compatible with a complete random stacking near the melting density, but the crystals tend towards fcc when either increasing or decreasing the overall volume fraction of the colloids. This work was extended by Poon and Pusey, ${ }^{7}$ where, among other things, the structure of the crystals was followed in time. They found that in an initially random-stacked sample (near the melting density), a fcc feature [i.e., a peak corresponding to the (200) line of fcc] develops in time. Comparable results were found in charged colloids, where the initially random-stacked structure tends towards $\alpha \approx 0.75$, named "faulted twinned fcc" by the authors. ${ }^{8}$ In an experiment under microgravity by Zhu et al., ${ }^{9}$ however, the crystals of hard colloidal spheres retain a random stacking for several days, without any evidence of a growing fcc feature. They therefore conclude that gravity stress promotes fcc, but the question regarding the equilibrium structure near the melting density of experimental "hard sphere" model systems when gravity is negligible is still open.

The question of the thermodynamically stable stacking in crystals of hard spheres was for the first time addressed by Frenkel and Ladd ${ }^{10}$ using computer simulation. Extension of this work reveals that fcc is significantly stable relative to hcp (Ref. 11) [though not as stable as claimed in (Ref. 12], but also relative to rhcp. ${ }^{13}$ As shown very recently by Mau and Huse, ${ }^{14}$ it is even stable relative to all possible stackings that are different from $\alpha=1$. However, the differences in entropy between the different stackings are extremely small, in particular near the melting transition where it is found to be on the order of $10^{-4} \mathrm{k}$ per particle (where $k$ is Boltzmann's constant). Because of the driving force towards fcc being so small, the systems may easily get trapped into metastable states, and different initial stackings in the crystals may lead to different structural evolution scenarios. On the 
other hand, subtle effects, such as polydispersity, a slight "softness" in the pair potential between the colloidal particles, or, indeed, stress induced by gravity, are expected to be important factors that determine the ultimate stacking in experimental systems of (nearly) hard colloidal spheres, as it is conceivable that they may even change the sign of the (very small) differences in entropy between the different stackings.

In this work we address the question as to how the structure of colloidal hard sphere crystals develop in time when gravity effects are minimal, polydispersity is small $(<3 \%)$, and the initial stacking in a single system is varied by applying different forms of shear stress.

\section{EXPERIMENT}

\section{A. Colloidal system}

The colloidal system, poly(methyl methacrylate) (PMMA), stabilized by the covalently surface linked stabilizer poly(12 hydroxy stearic acid) (PHS), was prepared according to the procedure described in Refs. 15 and 16. The radius of the particles (in the solvent mixture described below) is $470 \mathrm{~nm}$, and the polydispersity, estimated from light scattering and electron microscopy, is smaller than 3\%. Such a small polydispersity is only possible for relatively large particles. The particles were dispersed in a solvent mixture of (almost) equal refractive index and mass density as the particles; cis-decalin, tetralin and carbontetrachloride with mass fractions of $0.0493,0.3756$, and 0.5751 , respectively. The refractive index of this mixture is 1.5030 (for a wavelength of $589 \mathrm{~nm}$ ), and the mass density is $1.2381 \mathrm{~g} / \mathrm{cm}^{3}$ (at $293 \mathrm{~K}$ ), being significantly larger than the density of the PMMA particles in hydrocarbons such as dodecane, where it is found to be $1.18 \mathrm{~g} / \mathrm{cm}^{3}$. This difference is due to the fact that the particles were found to swell significantly in the mixture, i.e., a factor of 1.28 in diameter relative to the situation in dodecane, as determined by static light scattering and scaling on the freezing concentration. Because of this swelling, the solvent composition that corresponds to a minimum in the buoyant mass of the particles had to be determined by iteratively measuring the sedimentation velocity of the particles (using centrifugation), and adding one of two stock solutions of equal refractive index but different mass density. With this procedure, the density difference between particles and solvent was estimated to be of order $10^{-4}\left(\mathrm{~g} / \mathrm{cm}^{3}\right)$, as deduced from the sedimentation rate of the diluted particles in a sample with a somewhat mismatched refractive index. This density difference corresponds to a gravitational length $h$ $=k T / m_{b} g$ of order millimeters. In this equation, $k T$ is the thermal energy, $m_{b}$ the buoyant mass of a particle, and $g$ is the acceleration due to gravity. "Conventional" solvents for these particles, such as mixtures of decalin and tetralin, have densities of approximately $0.3 \mathrm{~g} / \mathrm{cm}^{3}$ smaller than the particles. For the particles of the size used here, this corresponds to $h$ of order micrometers. Systems under microgravity on the other hand, as in Ref. 9, have $h$ of order meters. So, the system studied here is just in between these two extremes. Note that the gravitational length $h$ is inversely proportional to the density difference between the colloids and the sol- vent, but inversely proportional to the cube of the size of the particles, i.e., $m_{b}=\pi D^{3} \Delta \rho / 6$, with $D$ the particle diameter and $\Delta \rho$ the mass density difference between colloids and solvent. So one could achieve a similar gravitational length by using "conventional" solvents and colloids smaller than approximately $100 \mathrm{~nm}$ in diameter. With these small particles, even true microgravity conditions ( $h$ of order meters) can be reached using the solvent mixture as described above. However, such small particles are significantly more polydisperse than large ones, with usually more than $5 \%$ polydispersity. As discussed in the Introduction, these large polydispersities may influence the equilibrium structure of the hardsphere crystal. It was verified that the system behaves as hard spheres by several observations. The most important is that the position of the first peak of the pair correlation function in a system of similar particles with a fluorescent core, as determined by confocal scanning laser microscopy, does not depend on the volume fraction. ${ }^{17}$ The range of volume fractions over which the pair correlation function was measured is $0.45-0.64$. This observation indicates that there is no significant soft repulsive part in the interaction potential. The possibility of an attractive contribution was excluded by measuring the value of the second virial coefficient using light scattering of similar, but smaller, dilute particles. This value equals the one for hard spheres. Another indication of hard sphere behavior is that is was found that the crystal volume in the coexistence region between colloidal fluid and crystal grows linearly with the (dry) mass fraction of colloid in the sample, and once the freezing volume fraction is set at 0.494 , the melting fraction follows being $0.543 \pm 0.011$, in good agreement (albeit with a large uncertainty) with the value of 0.545 as determined using Monte Carlo computer simulation of hard spheres. ${ }^{18}$

\section{B. Sample treatment}

The volume fraction of the sample that we studied is 0.55 , which approximately corresponds to the density where the nucleation rate is maximal. ${ }^{19,20}$ Before starting an experiment, the crystals that were formed were shear melted by gently tumbling the samples for at least several hours. Subsequently, nucleation took place under three different conditions, designated as I, II, and III, i.e.,

(I) Without any perturbation, in the sample holder of the scattering setup.

(II) During centrifugation with a speed corresponding to a gravitational length of approximately $h=10 \mu \mathrm{m}$, for $48 \mathrm{~h}$. After that, the sample was placed in the sample holder of the scattering setup, where the crystals that were formed during centrifugation partially melted due to convection induced by the difference in temperature between the sample and the thermostatted bath. Subsequently, the partly melted crystals grew again.

(III) Under manually applied oscillatory shear, induced by rotating the sample hence and forth with a frequency of approximately $1 \mathrm{~Hz}$. This treatment lasted for about $1 \mathrm{~h}$ in the sample holder of the scattering setup. 
It turns out that these conditions induce a significantly different stacking of the hexagonal layers that make up the colloidal crystals, as will be discussed in the next section.

\section{Scattering setup}

The scattering setup is described in detail in Ref. 21, and here it is only briefly summarized. A high angular resolution $\left(0.008^{\circ}\right)$ is achieved by using a diode array camera that is positioned in the focal circle of the light scattered from the sample, and around a cylindrical thermostating bath. The thermostating liquid (toluene) is almost refractive index matched with the dispersion. The total scattering angle range of the camera is $4.0^{\circ}$, and the full scattering pattern of interest (with a width of approximately $8^{\circ}-9^{\circ}$ ) was obtained by moving the camera along the focal circle. The sample was contained in a cylindrical cuvette with a volume of $20 \mathrm{ml}$, and the scattering volume (after expanding the beam of a 1 $\mathrm{mW} \mathrm{He} / \mathrm{Ne}$ laser) was approximately $1 \mathrm{~cm}^{3}$. This volume contains many (orientations of) crystallites on the order of $0.1 \mathrm{~mm}$ in size. It is interesting to note that in a sample close to the freezing volume fraction, indeed millimeter-sized crystals were found, as in Ref. 9. The scattering patterns were obtained by averaging 500 scans of $0.3 \mathrm{~s}$. During the accumulation of the scans the sample was gently rotated, so that a true light-scattering equivalent of a powder crystallographic pattern was obtained.

The structure factor of the crystals, $S(K)$, is ultimately obtained by dividing the scattered intensity, $I(K)$ by the measured single particle form factor, $P(K)$, as $I(K)$ $=P(K) S(K)$ in orientationally invariant materials, such as a powder of crystallites. As noted by Pusey et al. ${ }^{6}$ the data at scattering vector $K$ significantly beyond the (200) reflection of fcc indeed become unreliable as this is where a (steep) minimum of the form factor is found.

\section{Calculation of the powder diffraction pattern}

The reciprocal-space structure of the close-packed hexagonal crystal layers that were stacked in a manner defined by the probability $\alpha$ as defined in the introduction was computed $^{22}$ on the basis of Eqs. (9) and (17) from Loose and Ackerson. ${ }^{23}$ Powder averaging was carried out by numerical integration using Simpson's Rule. A Gaussian distribution of the size of cubic crystals with a width of $10 \%$ was assumed. Deviation from the cubic shape and the width of the size distribution does not significantly affect the ratios of the peak intensities. The size of the crystals mainly alters the broadness of the scattering peaks. Within the fixed cubic shape of the crystals, the size only has a relative significance, as hardly any difference in the scattering pattern is found even when the crystals are made twice as large in the direction of the hexagonal layers. Free parameters in the calculation are an overall scaling factor, the value of $\alpha$, and the average size of the crystals. Only the value of $\alpha$ significantly (and sensitively) affects the shape of the scattering pattern. The average size of the crystals was adjusted until the width of the (111)-Bragg reflection agreed as good as possible with the experimental width.

\section{RESULTS}

The powder patterns, at different times after nucleation, are shown in Figs. 1(a)-1(c) for the conditions as listed under (I) in the Experiment. Figures 2(a)-2(c) are the powder patterns of the system under conditions designated by (II), and Figs. 3(a) and 3(b) correspond to the conditions named (III).

The experimental pattern of the system that was nucleated under undisturbed conditions, $16 \mathrm{~h}$ after the nucleation process was initiated [see Fig. 1(a)] is well described by a purely random stacking of hexagonal layers, $\alpha=0.5$. A sharp main peak is present, with on both sides broad bands of diffuse scattering. The main peak is the (111)-Bragg reflection at $\left(K a_{\mathrm{fcc}} / 2 \pi\right)^{2}=3.0$, with $a_{\mathrm{fcc}}$ the size of the fcc unit cell. The shallow "prepeak" at $\left(K a_{\mathrm{fcc}} / 2 \pi\right)^{2}=2.67$ corresponds to the hcp (100) reflection, and the broad peak beyond the main reflection is at the same $K$ vector as the hcp (101) reflection at $\left(K a_{\mathrm{fcc}} / 2 \pi\right)^{2}=3.42$. After 2 weeks, however, another feature becomes apparent corresponding to the (200) line of a fcc structure, with $\left(K a_{\mathrm{fcc}} / 2 \pi\right)^{2}=4.00$. This pattern is shown in Fig. 1(b). This feature slowly grows in time, as can be seen from the pattern, in Fig. 1(c), that was measured 40 days after nucleation was initiated. The patterns in Figs. 1(b) and 1(c) could not be described by a single value of $\alpha$. Instead, a distribution of crystals with different stacking probabilities had to be assumed. This distribution is peaked at $\alpha=0.5$ and $\alpha=0.8$, details are given in the figure captions. As can be seen from Figs. 1(b) and 1(c), such a distribution describes the powder patterns rather well, although there is a systematic deviation at high scattering vectors in all the Figs. 1(a) -1 (c) and the calculated intensity of the main (111)-Bragg reflection tends to be too high. In order to illustrate that the calculated pattern is quite sensitive to the location of the peaks of the stacking probability distribution, we also show a pattern, in Fig. 1(c), that was calculated after forcing the distribution to be peaked at $\alpha=1.0$, and the weights of the other stacking probabilities were adjusted so as to get as good an agreement as possible with the data.

The powder pattern of the crystals that were nucleated under "normal" gravity conditions, subsequently melted in part and again grown [condition (II)], is shown in Figs. 2(a)2(c). The feature corresponding to the fcc (200) line is now overwhelmingly present even very shortly after nucleation [Fig. 2(a)]. Again, the scattering pattern could not be described by a single value of $\alpha$, and a distribution of crystals of different scattering probabilities had to be assumed (see figure captions). At short times after nucleation [Fig. 2(a)], the part of the scattering pattern around the fcc (200) feature is described well by theory, but in particular the part around the prepeak corresponding to hcp (100), is not. Better agreement is found when different crystal sizes are assumed, but we choose not to extend parameter space by this assumption as we have no independent proof of this really being the case. We therefore speculate that crystals of different size and/or shape, possibly in combination with defects, cause the deviation from theory in Fig. 2(a). This speculation may be verified by direct numerical computation of the structure factor of either computer generated crystals or experimentally obtained coordinates. At longer times, agreement with theory 

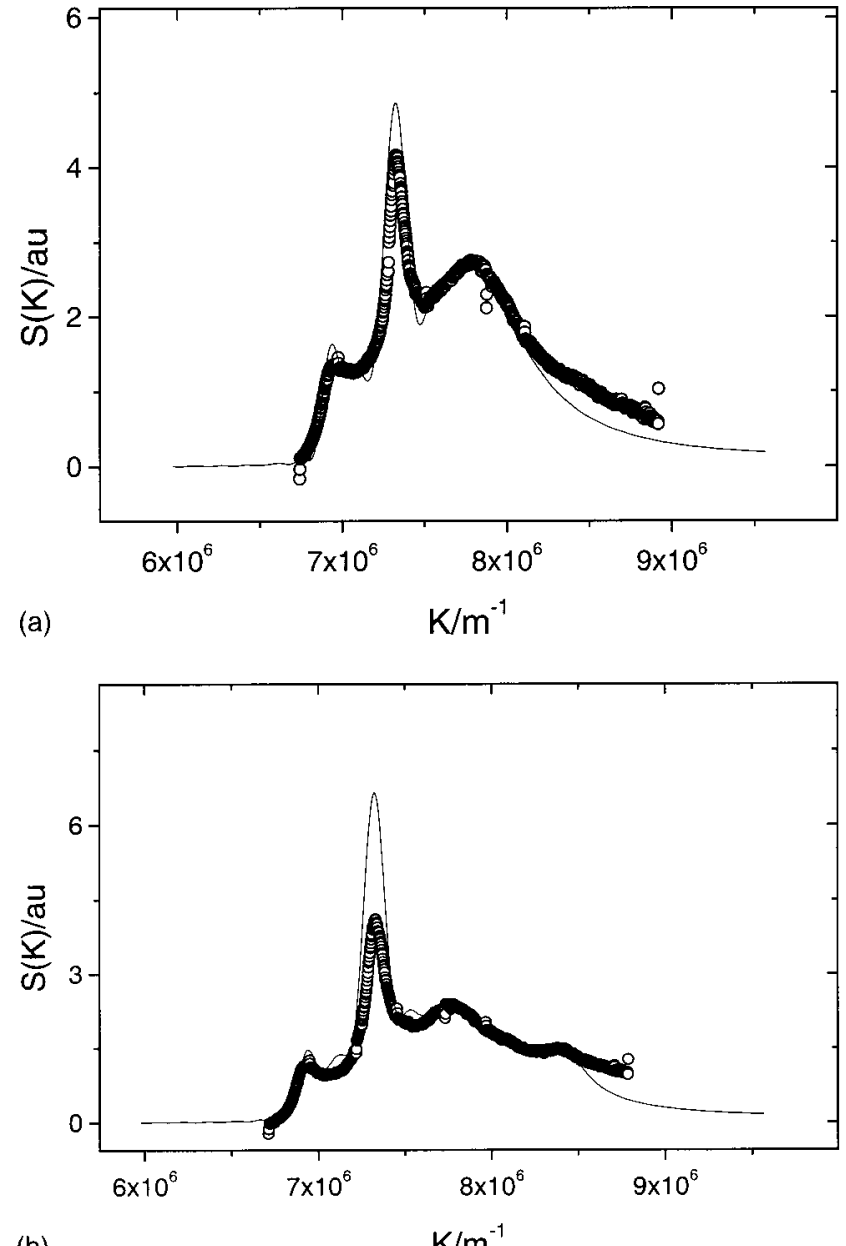

(b)

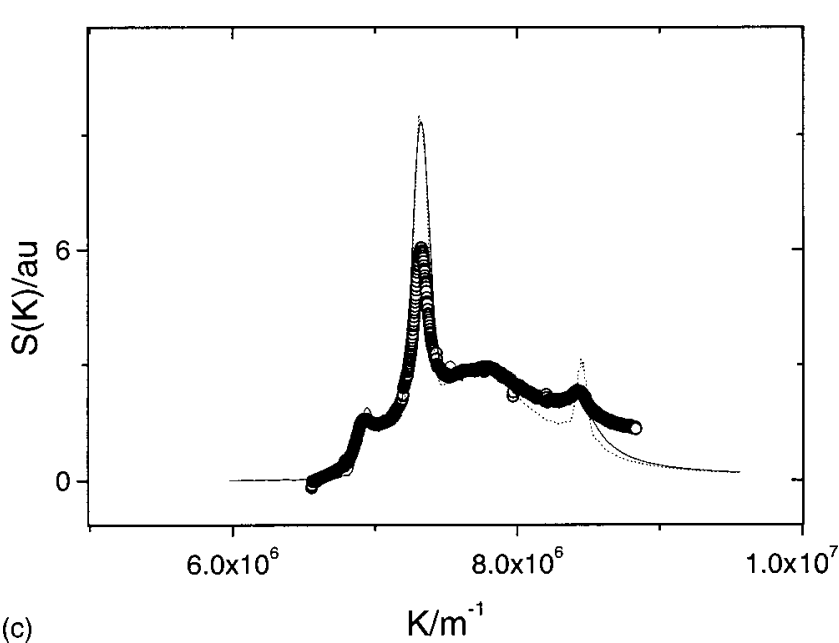

(c)

$$
\mathrm{K} / \mathrm{m}^{-1}
$$

FIG. 1. (a) Structure factor of colloidal hard spheres (PMMA-PHS) with volume fraction $\varphi=0.55$, and radius $R=470 \mathrm{~nm}, 16 \mathrm{~h}$ after nucleation under "milligravity" conditions and no applied shear [conditions listed under (I) in the Experiment]. Points are experiments, line is theory with cubic crystals of $10^{5}$ particles and $\alpha=0.5$. (b) Same sample as in (a), 13 days later. The line corresponds to a "best fit" to a distribution of crystallites of 1.25 $\times 10^{5}$ particles with different stacking probabilities with the following weights: $0.55(\alpha=0.5), 0.05(\alpha=0.6), 0.1 \quad(\alpha=0.7), 0.20 \quad(\alpha=0.8), 0.1 \quad(\alpha$ $=0.9$ ). (c) Sample as in (a), 40 days after nucleation. The solid line corresponds to a distribution of crystallites with stacking probabilities with weights, $0.45(\alpha=0.5), 0.1(\alpha=0.6,0.7), 0.25(\alpha=0.8)$, and $0.1(\alpha=0.9)$. The dashed line corresponds to a distribution that is forced to be peaked at $\alpha=1.0$, and the weights of the other stacking probabilities were adjusted to get a "best fit" to the data. The results are $0.45(\alpha=0.5), 0.1(\alpha=0.6), 0.05$ $(\alpha=0.7,0.8), 0.1(\alpha=0.9), 0.25(\alpha=1.0)$. Same crystal size as in (b).
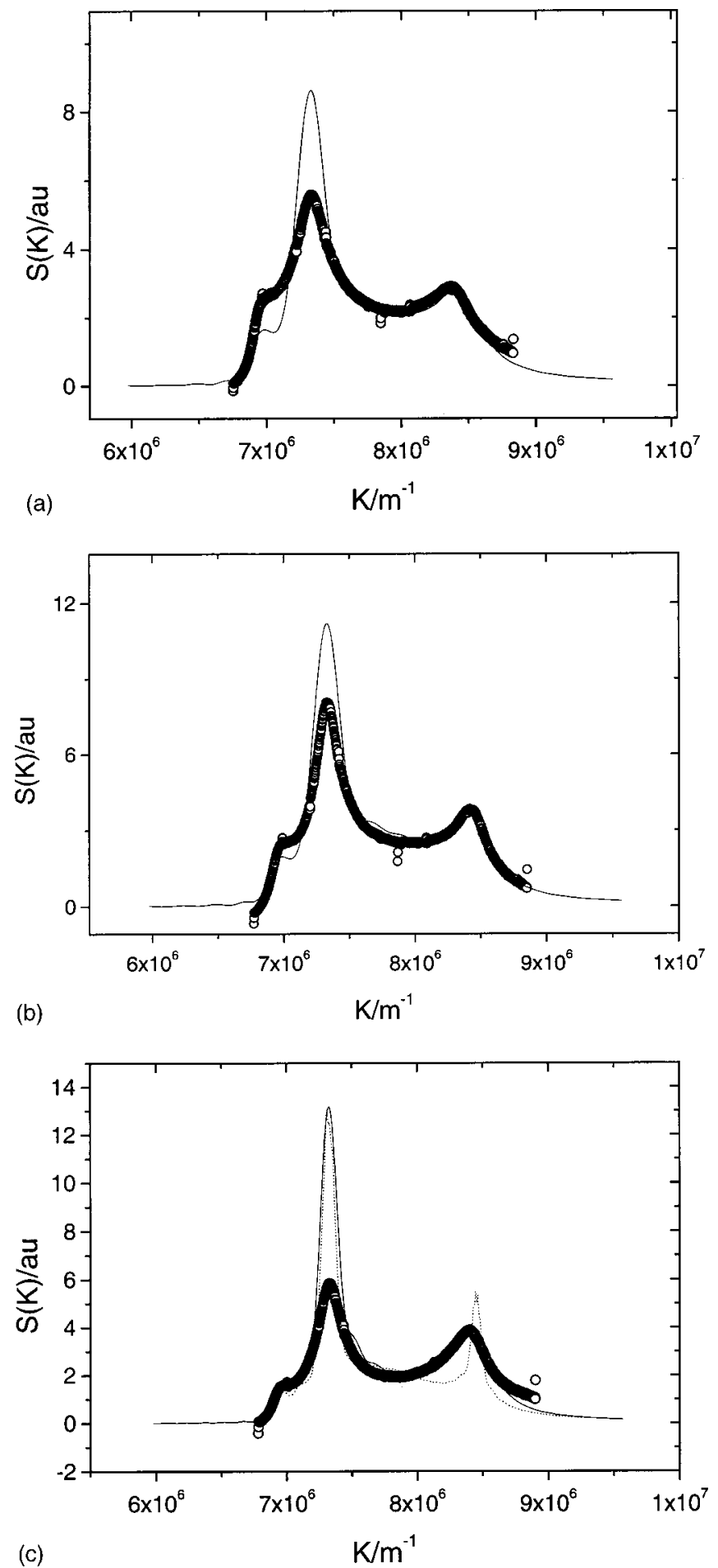

FIG. 2. (a) Sample (same as in previous figure) that was shear melted, centrifuged (with a speed corresponding to a gravitational length $h$ $=10 \mu \mathrm{m})$ for $48 \mathrm{~h}$, and subsequently placed in the sample holder, where (due to the difference in temperature) the crystals that were formed during centrifugation, partially melted. These conditions are listed under (II) in the Experiment. The scattering pattern was taken $20 \mathrm{~h}$ after the sample was placed in the scattering setup. The line corresponds to a distribution of stacking probabilities with weights $0.2(\alpha=0.5), 0.1(\alpha=0.6), 0.15(\alpha=0.7)$, $0.50(\alpha=0.8), 0.05(\alpha=0.9)$. Crystals contain $2.7 \times 10^{4}$ spheres. (b) As (a), 14 days later. Lines are theory with $0.20(\alpha=0.5), 0.05(\alpha=0.6), 0.1(\alpha$ $=0.7), 0.55(\alpha=0.8), 0.1(\alpha=0.9)$. Same crystal size as in previous figure. (c) Same sample 28 days after the pattern in (a) was taken. Solid line, 0.10 $(\alpha=0.5), 0.10(\alpha=0.6), 0.10(\alpha=0.7), 0.70(\alpha=0.8)$; crystals contain 1.25 $\times 10^{5}$ spheres. The dashed line corresponds to a distribution that was forced to be peaked at $\alpha=1.0$ [in the same way as in Fig. 1(c)]: $0.20(\alpha=0.5), 0.10$ $(\alpha=0.6-0.8), 0.15(\alpha=0.9), 0.35(\alpha=1.0)$. 

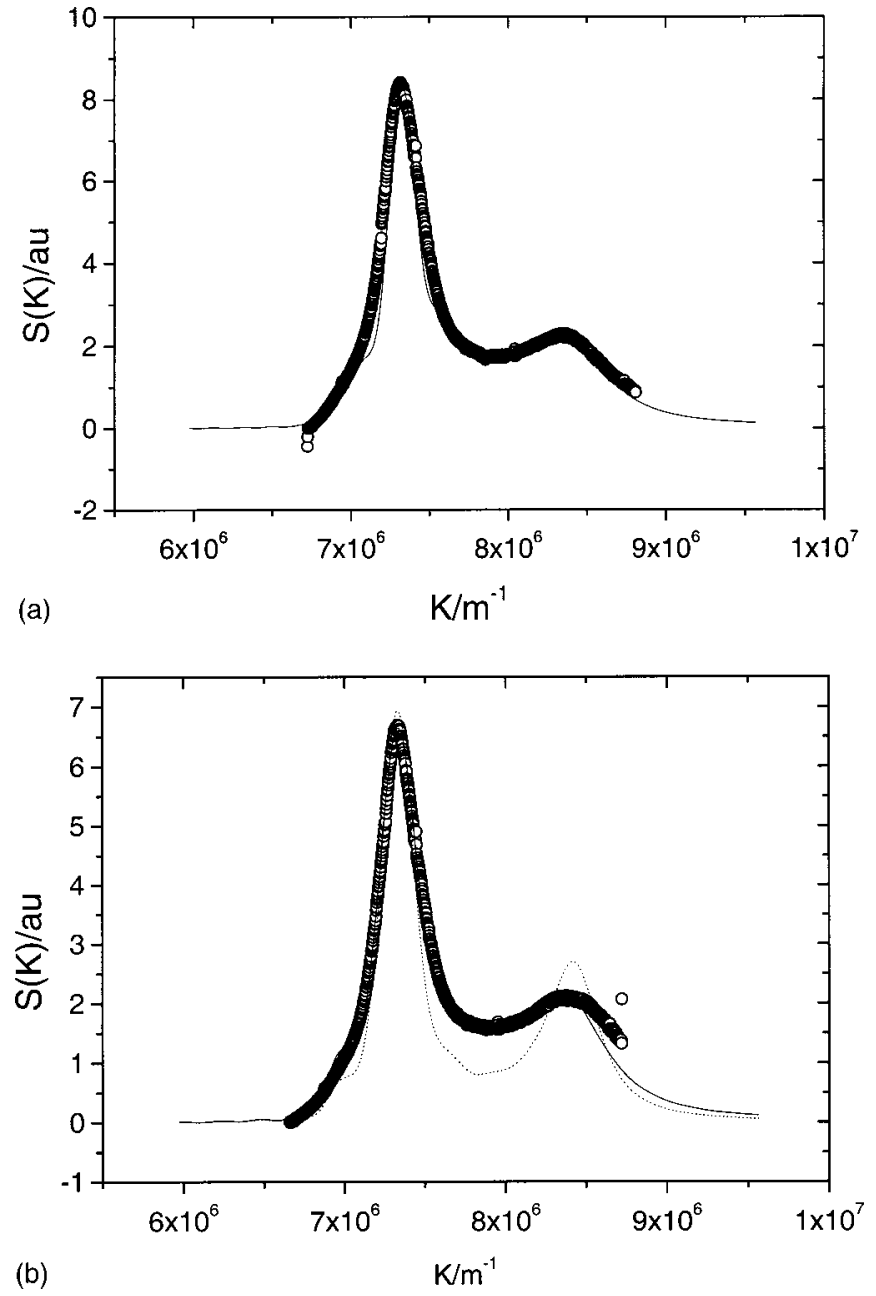

FIG. 3. (a) Sample (same system as in previous figures) that was shear melted, and subsequently gently sheared during nucleation for about $1 \mathrm{~h}$ by manually rotating the sample hence and forth with a frequency of approximately $1 \mathrm{~Hz}$ [conditions (III) in the Experiment]. The scattering pattern was taken $20 \mathrm{~h}$ after this treatment. Line corresponds to a single stacking probability with $\alpha=0.70$. Crystals contain $6.4 \times 10^{4}$ hard spheres. (b) As Fig. 3(a), 24 days later. Solid line has $\alpha=0.70$; dashed line (for comparison) has $\alpha=0.80$.

becomes better and, just as the sample at condition (I), the results indicate an increasing fraction of crystallites with $\alpha \approx 0.8$ that grows at the expense of the fraction of randomly stacked crystals $(\alpha=0.5)$. As shown in Fig. 2(c), the experimental results are poorly described by theory if a growing fraction of pure $(\alpha=1.0)$ fcc crystals is assumed.

The results on the system that was gently sheared during nucleation [situation (III)] are shown in Figs. 3(a) and 3(b). Just as under condition (II), the fcc (200) feature is already significantly present at relatively short times after nucleation. In this case, the powder pattern is rather well described by theory using a single value of the stacking probability, $\alpha=0.70$, although the (indeed only very weakly present) prepeak at $K$ corresponding to the hcp (100) reflection is not seen in the experiment. Clearly [Fig. 3(b)] the situation does not significantly change in 24 days.

\section{DISCUSSION}

It follows from Figs. 1(a)-1(c) that a system of colloidal hard spheres, of volume fraction 0.55 , that is left undisturbed during nucleation, initially forms a purely random stacked crystal of hexagonal layers. This result is in complete agreement with Ref. 6, and also with the observation in microgravity, ${ }^{9}$ although in the latter case only qualitative conclusions can be drawn. After 2 weeks, a small but significant fraction of crystallites are transformed into a stacking that can be termed "faulted-twinned fcc", 8 i.e., a stacking like $A B C B C A C B A C B A \ldots$, with $\alpha=0.8$, and this fraction keeps growing in time up to at least 40 days after nucleation. In a study by Poon and Pusey, ${ }^{7}$ is was observed that at a volume fraction comparable with the one used here, initially a random stacked (with $\alpha=0.5$ ) crystal forms, but the fcc (200) feature develops within a day. ${ }^{24}$ The colloidal particles used in Ref. 7 are about twice as small as the ones studied here. At comparable volume fractions, the relevant (and relative) timescale in the two systems is the Brownian time, i.e., the time it takes for a particle, at infinite dilution, to diffuse over its own radius $R, \tau=R^{2} / 6 D_{0}$, with $D_{0}$ the diffusion coefficient at infinite dilution. As the solvent viscosities are comparable we have $D_{0} \sim 1 / R$, implying that our system is roughly 8 times "slower" as the one studied in Ref. 7. Thus, on the Brownian time scale it takes at least twice as long in our system for the fcc feature to develop (after 1 week, no fcc feature is obvious; not shown). The only difference between the two systems, besides the particle radius, is the gravitational length of the particles, which in our system is on the order of millimeters, while in the one in Ref. 7 it is on the order of micrometers. So, gravitational stress indeed promotes fcc, as was concluded by Zhu et al. ${ }^{9}$ However, our results show that even when gravitational stress is very small, as quantified by the gravitational length, crystals still tend to transform into "faulted-twinned fcc," it only takes more (Brownian) time relative to systems under normal gravity conditions. This is probably the reason why the fcc feature was not seen in the space shuttle experiment. Indeed, Pronk and Frenkel ${ }^{13}$ recently computed the rate at which colloidal crystals of hard spheres are expected to transform from a random stacking into pure fcc. They show, based on free energy differences and a Wilson-Frenkel growth law, that such a transformation takes months rather than days in unperturbed systems, but the precise rate is quite sensitive to the size distribution of the crystals. In our system crystals with $\alpha=0.8$ grew instead of $\alpha=1.0$, and we did not look at the size distribution of the crystals. Therefore we cannot make a comparison with their predictions.

We attempted to simulate "normal" gravity conditions by centrifugation of the sample, but as described in the previous sections, the crystals that were formed during centrifugation partly melted when placed in the thermostatted sample holder of the scattering setup [treatment designated as (II) in the Experiment]. Nonetheless, the procedure leads to crystals that contain a much higher fraction of faultedtwinned fcc (with $\alpha=0.8$ ), even at the shortest times studied. Just as in the system after the previous treatment, this fraction grows in time, at the expense of the fraction with $\alpha=0.5$. Now in the last series of experiments [(II) in the Experiment] the sample was sheared manually, leading to a structure still different from the one resulting from the other treatments, i.e., a faulted-twinned fcc with $\alpha=0.7$, with no 
indications of the presence of random-stacked crystals. So the (shear) history of the sample sensitively determines the structure of the crystals. In general, we find that shear stress, either induced by centrifugation (or "gravity"), or by manually rotating the sample, tends to promote faulted-twinned fcc. However, in the first situation, a mixture of differently stacked crystals is found, with a small peak at $\alpha=0.5$ and a large one at $\alpha=0.8$, but in the second situation the experiments are best described by a single $\alpha=0.7$. It would be interesting to systematically, and quantitatively, investigate the influence of shear history on the structure of the crystals being formed. At this point we summarize our findings as follows: in crystals of colloidal hard spheres, a fraction of crystallites with $\alpha=0.8$ tends to grow. If crystals with a single $\alpha=0.7$ are generated, the structure does not change for a significant amount of time. Thus, the present observations rule out the possibility that a randomly stacked colloidal crystal is thermodynamically stable. Instead, either faultedtwinned fcc is the thermodynamically stable structure of colloidal hard-sphere crystals, or the system kinetically tends to get trapped into this structure. This question may be resolved by starting with crystals of pure fcc, and see if a fraction with smaller $\alpha$ grows. We were unable to generate pure fcc crystallites in this work, but perhaps such a structure can be grown on a template. ${ }^{25}$ As mentioned in the Introduction, computer simulations by Mau and Huse, ${ }^{14}$ addressing all possible stackings, demonstrate that pure fcc (with $\alpha=1.0$ ) has the highest entropy. This simulation takes into account interactions over up to five consecutive layers of spheres. At the melting density, interlayer entropic interactions are found to fall off very slowly compared to the situation at the closepacked density. This leaves the possibility, at least in principle, that faulted-twinned fcc, with $\alpha=0.7-0.8$, is ultimately stabilized by entropic interactions that are ranged beyond these five layers. However, especially at the melting density, the relative differences in entropy are very small (of order $10^{-4} k$ per sphere), so that long-lived metastable states are feasible even if fcc indeed proves to be the ultimately stable stacking of hard spheres at the melting density. Experiments in real-space, using confocal scanning laser microscopy, are currently initiated in order to learn more about the mechanism of the structural transitions that we observed in this work.

\section{ACKNOWLEDGMENTS}

We thank Stuart Henderson for kindly providing the light-scattering code. W.K.K. thanks Wilson Poon and Henck Lekkerkerker for enlightening discussions. In particular, Wilson Poon is thanked for pointing out that the data in Ref. 7 could not be described by a single value of $\alpha$. Alfons van Blaaderen and Daan Frenkel are thanked for a critical reading of the manuscript. We gratefully acknowledge the Royal Netherlands Academy of Arts and Sciences for providing a fellowship for W.K.K.

${ }^{1}$ W. W. Wood and J. D. Jacobsen, J. Chem. Phys. 27, 1207 (1957).

${ }^{2}$ B. J. Alder and T. E. Wainwright, J. Chem. Phys. 27, 1208 (1957).

${ }^{3}$ P. N. Pusey and W. van Megen, Nature (London) 320, 340 (1986).

${ }^{4}$ T. C. Hales, http://www.math.Isa.umich.edu/ hales/countdown.

${ }^{5}$ A. J. C. Wilson, X-Ray Optics, Methuen's Monographs on Physical Subjects, edited by B. L. Worsnop (Methuen, London, 1949).

${ }^{6}$ P. N. Pusey, W. van Megen, P. Bartlett, B. J. Ackerson, J. G. Rarity, and S. M. Underwood, Phys. Rev. Lett. 63, 2753 (1989).

${ }^{7}$ W. C. K. Poon and P. N. Pusey, in Observation, Prediction, and Simulation of Phase Transitions in Complex Fluids (Kluwer Academic, Varenna, 1994).

${ }^{8}$ C. Dux and H. Versmold, Phys. Rev. Lett. 78, 1811 (1997).

${ }^{9}$ J. Zhu, M. Li, R. Rogers, W. Meyer, H. Ottewill, Crew Shuttle Columbia, W. B. Russel, and P. M. Chaikin, Nature (London) 387, 883 (1997).

${ }^{10}$ D. Frenkel and A. J. C. Ladd, J. Chem. Phys. 81, 3188 (1984).

${ }^{11}$ P. G. Bolhuis, D. Frenkel, S-C. Mau, and D. A. Huse, Nature (London) 388, 235 (1997)

${ }^{12}$ L. V. Woodcock, Nature (London) 385, 141 (1997).

${ }^{13}$ S. Pronk and D. Frenkel, J. Chem. Phys. 110, 4589 (1999).

${ }^{14}$ S.-C. Mau and D. A. Huse, Phys. Rev. E 59, 4396 (1999).

${ }^{15}$ L. Antl, J. W. Goodwin, R. D. Hill, R. H. Ottewill, W. M. Owens, S. Papworth, and J. A. Waters, Colloid Surf. 17, 67 (1986).

${ }^{16}$ C. Pathmamanoharan, C. Slob, and H. N. W. Lekkerkerker, Colloid Polym. Sci. 267, 448 (1989).

${ }^{17}$ W. K. Kegel and A. van Blaaderen, Science 287, 290 (2000).

${ }^{18}$ W. G. Hoover and F. H. Ree, J. Chem. Phys. 49, 3609 (1968).

${ }^{19}$ J. S. van Duijneveldt and H. N. W. Lekkerkerker, in Science and Technology of Crystal Growth, edited by J. P. van der Eerden and O. S. L. Bruinsma (Kluwer Academic, Dordrecht, 1995).

${ }^{20}$ J. L. Harland, S. I. Henderson, S. M. Underwood, and W. van Megen, Phys. Rev. Lett. 75, 3572 (1995).

${ }^{21}$ J. K. G. Dhont, C. Smits, and H. N. W. Lekkerkerker, J. Colloid Interface Sci. 152, 386 (1992).

${ }^{22}$ S. I. Henderson, Polyxtal, a light-scattering code 1995 (unpublished).

${ }^{23}$ W. Loose and B. J. Ackerson, J. Chem. Phys. 101, 7211 (1994).

${ }^{24}$ W. C. K. Poon (personal communication).

${ }^{25}$ A. van Blaaderen, R. Ruel, and P. Wiltzius, Nature (London) 385, 321 (1997) 